E.L.U.A. 2, 1984, págs. $207-231$

\title{
FUNCIONES DE EXPRESIÓN, ORACIONES, ACTOS COMUNICATIVOS, TEXTOS (ASPECTOS DEL SIGNIFICADO Y DE SU TEMATIZACIÓN EN LA ESTRUCTURA DE UNA TEORÍA TEXTUAL)
}

\author{
JÁNOS S. PETÖFI \\ (Universidad de Bielefeld)
}

\section{Consideraciones previas}

Quiero explicar en este trabajo algunos aspectos del significado (significación) y de la interpretación del significado (construcción de significado) de objetos verbales de diferente complejidad y función.

El modelo de significado que sirve de base puede caracterizarse, en sus lineas principales, como sigue:

Todos los objetos $/ \mathrm{O} /$ utilizados/utilizables como signos simples o complejos son considerados como unidades de un significante (formativo $/ \mathrm{OF} /$ ) y de un significado. El significado representa un conjunto de dos elementos; uno es el correlato extralingüístico /OC/ (objeto o estado de cosas) que el objeto verbal que ha de ser interpretado, según opinión del intérprete, denota sistemática o comunicativamente, mientras que el otro elemento - también según la opinión del intérprete- es el conocimiento correspondiente sistemática o comunicativamente a este correlato (expresado de otro modo: el sentido /OS/ designado sistemática o comunicativamente por medio del significante). La relación entre un objeto verbal cualquiera, el componente de significante y el componente de significado de este objeto y un intérprete $I_{x}$ cualquiera está representada en el esquema 1. Sobre la base de esta explicación es evidente que no considero la significación como una propiedad inherente de los objetos verbales. Para un mismo objeto verbal pueden construirse diferentes significados en la mayoría de los casos. 


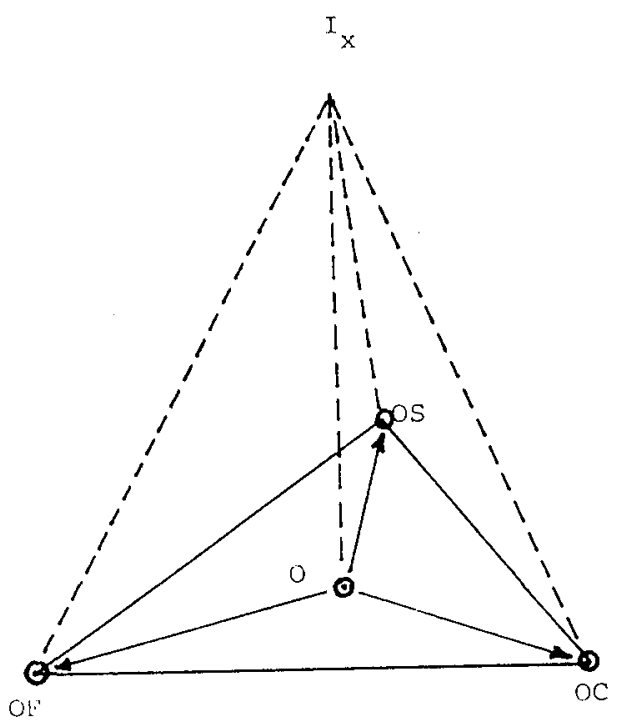

Esquema 1.-El triángulo semiótico. 
Para garantizar un adecuado análisis y descripción de la relación significante-significado considero necesaria la utilización de una armazón teórica (teórico-textual) que haga posible, mediante el uso de un thesaurus especificamente elaborado y de una lengua canónica, la construcción del significado de un objeto verbal de cualquier complejidad. El componente de thesaurus de una teoria textual ' dentro de cuya armazón analizaré aquí algunos aspectos del significado, debe constar de un subcomponente de léxico y de un subcomponente de enciclopedia (que opera con el léxico). El primero contiene el conocimiento relacionado con las clases de objetos y las clases de estados de cosas potenciales, el segundo sirve como almacén del conocimiento concerniente a objetos individuales y estados de cosas. El saber contenido en el léxico tiene que almacenar el conocimiento en forma de sistema de representaciones de explicación, mientras que la enciclopedia debe estar construida de tal manera que las explicaciones almacenadas en el léxico también sean utilizables para la explicación lingüistica del artículo de la enciclopedia ${ }^{2}$.

La lengua canónica opera con estructuras sobre las que puede aplicarse el principio de pregunta. Esto significa que el componente de significado de un constructo canónico de cualquier complejidad puede construirse a partir del componente de significado de sus constituyentes ${ }^{3}$.

Uso en este artículo las siguientes convenciones de signos: si el objeto verbal que hay que analizar es una palabra, una expresión o una oración, éstas se ofrecen sin subrayar y con comillas simples. Si es un acto comunicativo posible o un texto posible, se presenta subrayado y con comillas simples. Finalmente, si es un acto comunicativo efectivo, se ofrece subrayado y con comillas dobles. (En las secciones correspondientes se realiza una interpretación de estos términos.) El componente de significante de un objeto verbal es indicado por medio del subrayado y de las comillas especificas "'..'" "; el componente de correlato lo es mediante letras mayúsculas y las comillas especificas " "..."”; por último, el componente de sentido se indica con letras mayúsculas y con las comillas especificas «'...'».

Disponemos en español de varios trabajos en los que János S. Petöfi expone su teoría lingūístico-textual: Petöfi, 1979a, 1979b, 1979c, 1979d, 1979e. (N. del T.)

2 Para las cuestiones del thesaurus (léxico y enciclopedia), cfr. Petöfi, 1983; Neubauer-Petöfi, 1981; Putnam, 1978, y Stegmüller, 1979.

3 Vid. Biasci-Fritsche para las cuestiones de la lengua canónica. 


\section{Funciones de expresión en el léxico}

1.0. La noción función de expresión se utiliza en analogía con la noción función de proposición. Designo con ella las estructuras que contienen también variables, a partir de las cuales, mediante sustitución de las variables por constantes, pueden construirse expresiones. El término expresión designa aquí un constructo canónico y es neutro tanto en atención a los componentes semióticos como en relación con las funciones dentro de una estructura canónica compleja.

1.1. El léxico asigna funciones de expresión a las palabras y a las expresiones idiomáticas. La representación formal (1) describe la estructura canónica básica, esto es, en los niveles más abstractos, la cual permite la representación de todas las funciones de expresión, sin tener en cuenta su tipo semiótico, su categoría canónica y su complejidad.

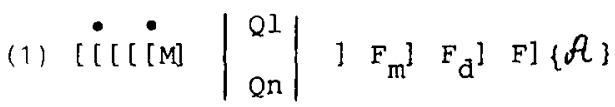

Los simbolos de (1) tienen las siguientes equivalencias: $\{A\}$, parte de argumento; la parte anterior a la parte de argumento es la parte de funtor. La parte de argumento es un conjunto de $n$ elementos, compuesto de pares < indicador de argumento, variable de argumento >, por lo que $n$ es un número natural dependiente de $F$. Son opcionales los elementos de la parte funtorial situados entre corchetes con un punto sobre el corchete de apertura. Los símbolos $M, Q I, Q n, F_{m}, F_{d}$ y $F$ indican la serie correspondiente a modificador (M), calificador (Ql), cuantificador $(Q n)$, unidad de medida $\left(F_{m}\right)$, dimensión $\left(F_{d}\right)$, asi como funtor (básico) (F).

Consideremos algunos ejemplos para la utilización de la representación formal (1) (cfr. esquema 2) ${ }^{4}$. Sin embargo, antes de una u otra lectura de estos ejemplos proporcionaremos algunas observaciones generales para el esquema 2:

4 Mantenemos el esquema 2 como aparece en el original alemán y ofrecemos la equivalencia española de las expresiones que en él hay. laüft: corre: geschw (Geschwindigkeit): rapidez; zehn: diez; groß: grande; schnell: rápido; sehr: muy. " $\mathrm{Km} / \mathrm{h}$ » indica kilómetros por hora. (N. del T.) 
(1)

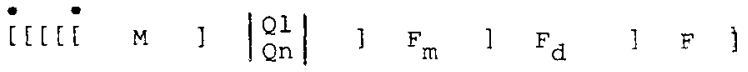

(2) $O_{1}:$ 'Iäuft'

\begin{tabular}{|c|c|c|c|c|c|}
\hline $\mathrm{OF}_{1}:{ }^{\top}$ läuft & {$[[][[]$} & 1 & ] & ] & ]$\leq$ läuf $\succ]\left[x_{1}: x\right\}$ \\
\hline$\propto_{1}:$ 'LALUET' & $i[0 ;$ & ] & ] & ] & $\underline{\underline{f} 17}]\left\{\mathrm{r}_{1}: \underline{x}\right\}$ \\
\hline 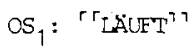 & {$[c][$} & ] & ] & ] & ] LAUFT $]\left\{r_{1}: x\right\}$ \\
\hline
\end{tabular}

(3) $\mathrm{O}_{2}:$ 'geschw'

\begin{tabular}{|c|c|c|c|c|c|c|}
\hline $\mathrm{OF}_{2}:{ }^{7}$ geschw' & $\dot{\theta}[\operatorname{ci}$ & 1 & ] & J 〈geschw> & & $I\{A\}$ \\
\hline$\alpha_{2}:{ }^{r} \mathrm{CESCHW}^{7}$ & {$[[][]$} & j & ] & $\underline{f}_{d} 8$ & & $1\{0\}\}$ \\
\hline$O S_{2}:{ }^{r}{ }^{r} G E S C H W^{\top}$ & {$[[\in]$} & I & ] & I GESCHW & $\perp$ & ]$\{\Omega\}$ \\
\hline
\end{tabular}

(4) $\mathrm{O}_{3}:{ }^{\prime} \mathrm{km} / \mathrm{h}$ '

$\mathrm{OF}_{3}: \quad \mathrm{ran} / \mathrm{n}^{\mathrm{r}}$

icisi

$k \underline{k n>}>\langle\underline{\langle}\rangle]$

I\{A\}

$\propto_{3}: \quad \mathrm{mM} / \mathrm{H}^{1}$

] $\in_{-\pi} 21 \quad$ ।

I $\{$ R\}

$\mathrm{OS}_{3}: r_{\mathrm{KM} / \mathrm{H}^{\top} \mathrm{T}}$

i $[i[i$

] $\mathrm{KM} / \mathrm{H} \quad$ ]

(d) 3

I\{A\}

(5) $O_{4}:$ 'zehn'

$\mathrm{OE}_{4}:$ 'DO;zehn ${ }^{\mathrm{r}}$ [ [ [ [ [

$\infty_{4}:{ }_{10}{ }_{10} \mathrm{ZEHN}^{\top} \quad$ [ [ [ [ [ [

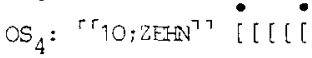

] $\langle 10\rangle]$

J $\{Q\}$

] gn 18 ]

I $\{\Omega\}$

] $2 E+N$ ]

$1\{a\}$

(6) $O_{5}:$ 'groB'

$\mathrm{OF}_{5}$ : $^{\prime} \operatorname{grOB}^{\mathrm{r}}$

if [ [ :

] 〈groß> ]

]$\{A\}$

$\infty_{5}:$ 'GROB!

] gl135 ]

$\mathrm{OS}_{5}$; [TGROB'

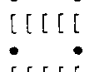

GPOB

] $]$

] $\{A\}$

]$\{A\}$

(7) $0_{6}:$ 'schnell'

$\mathrm{OF}_{6}:$ 'schnelir [ [ [ [ $\dot{0}$

] 〈scinel1) I

]$\{\mathcal{A}\}$

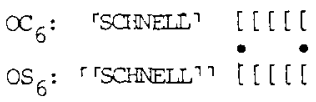

] g157

$1\{\AA\}$

I SCHNELE I $\longrightarrow$ ]

(d) I $1\{\Omega\}$

(8) $0_{7}:$ 'sehr'

$\mathrm{OF}_{7}:$ 'sehr' i[c[i sehr>

]$(A)$

$\propto_{7}:$ ISEHR ${ }^{7} \quad\left[\left[\left[\left[\left[\begin{array}{lll}{[} & \mathrm{m} 23\end{array}\right]\right.\right.\right.\right.$

] $\{\Omega\}$

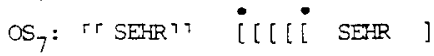

Esquema 2. 
- En relación con OF y OS hablo de representación /OFR, OSR/, y en relación con $\mathrm{OC}$ hablo de indicador /OCl/ (para evitar las conocidas dificultades, relativas a la extensión, de la definición de significado de Putnam).

- Como indicadores de correlato uso símbolos que consisten en combinaciones de letras y de cifras.

- Los paréntesis angulares en la representación OF indican que en ella está contenido un formativo (una serie ordenada de letras o de sonidos).

- La $d$ inscrita en un círculo en las representaciones de $\mathrm{OS}_{3}$ y $\mathrm{OS}_{6}$ indica que los dos sentidos "KM/H" y "SCHNELL" que hay en cuestión implican dimensión futura. (Esta dimensión es en ambos casos la rapidez.)

— El signo “__ en la representación de $\mathrm{OS}_{5}$ y $\mathrm{OS}_{6}$ indica que los dos sentidos "GROSS" y "SCHNELL" contienen también de modo implicito un valor $F_{m}$.

Consideremos como ilustración para los ejemplos ofrecidos en el esquema las lecturas de (2) y (3). Junto a mis argumentos en estas lecturas tomo como base el uso lingüístico de una comunidad lingüística dada y los conocimientos válidos (estereotipos) en el interior de esta comunidad.

La representación de (2) se lee como sigue:

Si para el objeto señalado con el símbolo $\underline{x}$ en la parte de argumento "A» es válida la relación $\underline{\mathrm{f}} 17$, entonces los miembros de la comunidad lingüística dada dicen en el contexto normal que este objeto 'corre' ('läuft') (denotación).

Si para el objeto señalado con el símbolo $x$ en la parte de argumento "A» la relación f 17 es válida, entonces los miembros de la comunidad lingüistica dada disponen en el contexto normal, a propósito de esta relación, del conocimiento de que este objeto corre (correspondencia).

La designación puede, sobre la base de estas dos explicaciones, ser formulada claramente: si los miembros de la comunidad lingüistica dada afirman, sobre el objeto señalado con el símbolo $\underline{x}$, que 'corre', designan entonces su conocimiento sobre el estado de cosas 'OBJETO x CORRE' ('OBJEKT x LÄUFT').

La representación de (3) ha de leerse como sigue:

Si está especificada la relación válida para el objeto (u objetos) señalado en la parte de argumento "A $A$ a propósito de la dimensión $\underline{\mathrm{f}} 8$, entonces los miembros de la comunidad lingüística dada dicen en el contexto normal que esta dimensión es la 'rapidez' ('Geschwindigkeit') (denotación). 
Si la relación válida para el objeto (u objetos) señalado en la parte de argumento «A» a propósito de la dimensión f8 está especificada, los miembros de la comunidad lingüística dada disponen en el contexto normal, en relación con esta dimensión, del conocimiento de que esto es la rapidez (correspondencia).

Me gustaria renunciar aquí a una formulación de la relación de designación.

Las relaciones semióticas concernientes a los ejemplos anteriores pueden ser formuladas de modo análogo a las arriba presentadas".

1.2. Para el formativo, el sentido y el correlato pueden ser interpretadas ulteriores operaciones. Nombrémoslas ordenadamente: expansión/contracción, explicación/condensación y análisis/síntesis. (Ya que tanto la representación de formativo y sentido como el indicador de correlato son configuraciones de símbolos, es conveniente hablar de expansión/contracción igualmente en los tres casos de representación.)

Para estas operaciones puede decirse brevemente en relación con palabras/giros lo siguiente:

En la percepción de un objeto/estado de cosas conocemos este objeto/este estado de cosas casi siempre como un correlato integrado por un haz de propiedades si y verdaderamente sólo si percibimos las propiedades particulares en el interior de este haz no conocido particularmente. Denotamos este correlato mediante una palabra simple o compuesta, o mediante un giro (como formativo), y consideramos este formativo en primer lugar como designador de un sentido global. Asi se origina, por ejemplo, el conjunto de tres elementos 'corre', 'CORRE', "CORRE" ('läuft', 'LÄUFT', "LÄUFT"'). (Los elementos obtenidos por esta vía están señalados en el esquema 3 con los símbolos OF, OC y OS.)

No obstante, si resulta preciso, podemos analizar los correlatos particulares, es decir, podemos separar, sobre el nivel de la percepción o del análisis instrumental, sus componentes/propiedades particulares, unos de otros. En este análisis podemos penetrar en las zonas profundas tanto como nuestros órganos de sentido o nuestros medios de análisis lo permitan. Podemos entonces denotar particularmente mediante formativos los componentes/propiedades que creemos haber constatado en el análisis, y podemos producir la configuración del componente de sentido designado a través de estos formativos. Así llegamos finalmente a un correlato analizado, a un formativo expandido

\footnotetext{
5 Para ulterior análisis de la fórmula (1), cfr. Heydrich-Petöfi, 1983.
} 


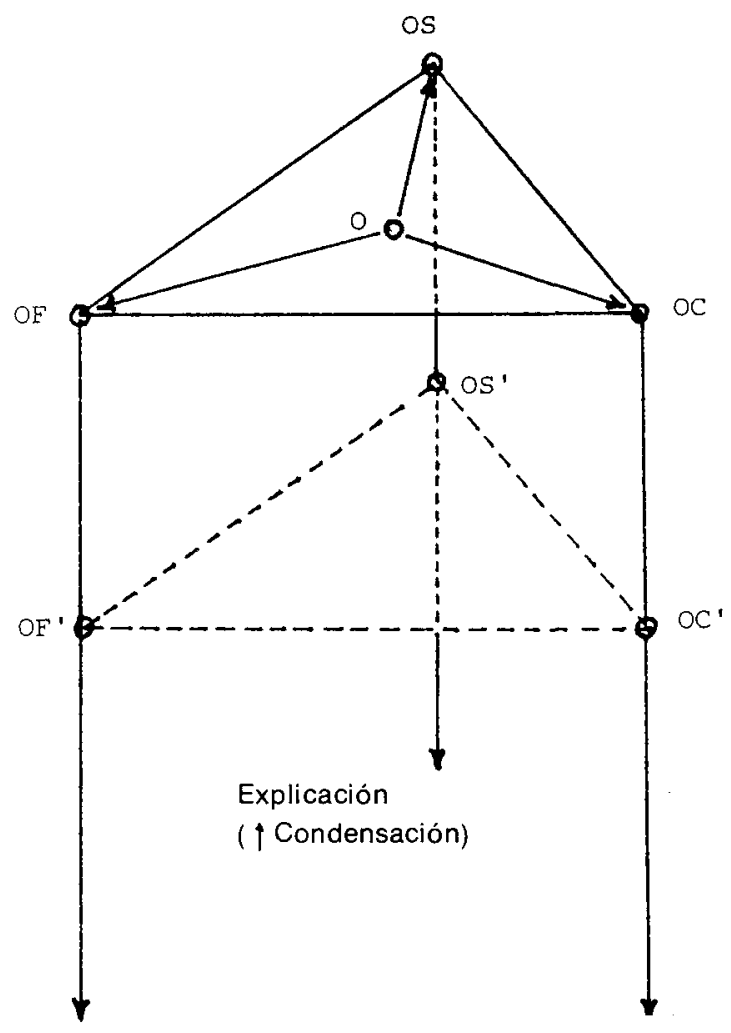

Expansión

Análisis

( $\uparrow$ Contracción)

( $\uparrow$ Sintesis)

Esquema 3.-Operaciones realizables por medio de los componentes semióticos. 
y a un sentido explícito. (Los elementos obtenidos por esta vía están señalados en el esquema 3 mediante los simbolos “OC'», “OF'» y "OS'».)

En las ciencias formales, así como en la clasificación de objetosestados de cosas, podemos, no obstante, proceder en general también especulativamente, esto es, podemos añadir a los sentidos globales concretos más o menos explicaciones cualesquiera, y podemos, con la ayuda de éstas, construir clases de objetos y de estados de cosas.

Por lo que se refiere a las relaciones de denotación, designación y correspondencia, a partir de los elementos

$\begin{array}{lll}\text { OF } & \text { OS } & \text { OC } \\ \text { OF }^{\prime} & \text { OS } & \text { OC' }\end{array}$

podemos construir así todos los triángulos semióticos posibles (esto es, ocho), e interpretar las tres relaciones a propósito de los ocho triángulos semióticos. (De hecho, realizamos esto en la praxis de la comunicación cotidiana.)

1.3. La tarea principal en la elaboración del léxico es la construcción de las explicaciones.

Antes de todo debemos distinguir entre los miembros no expertos y los diferentes grupos de expertos de la comunidad lingüística. En relación con un mismo objeto/estado de cosas pueden igualmente los no expertos y los diferentes expertos disponer de conjuntos de conocimientos diferenciados, y en la mayoría de los casos disponen de ellos. Es conveniente representar las explicaciones que corresponden a estos conjuntos de conocimientos como sistemas diferenciados, puestos en relación, no obstante, unos con otros.

Es conveniente declarar, dentro de los sistemas de explicación particulares, determinados sentidos concretos como atómicos (primitivos), y construir las explicaciones con ayuda de estos sentidos atómicos. Naturalmente, no es necesario esperar de todas las explicaciones que estén construidas exclusivamente con primitivos. Sin embargo, es importante exigir que la explicación de un sentido no atómico pueda ser reducida, en una cifra final de acciones, a una explicación que contenga exclusivamente los primitivos. Me gustaría poner de relieve que aqui se trata de la declaración de primitivos - como consecuencia de la convicción de que los sentidos atómicos no son elementos que puedan ser hallados con ayuda de un procedimiento exacto correspondiente. La declaración como procedimiento también significa, por otra parte, que incluso dentro del mismo sistema de explicaciones diferen- 
tes conjuntos de sentido pueden ser declarados primitivos. Esto, teóricamente, no es ningún problema; tiene solamente la consecuencia práctica de que los correlatos dentro de un sistema sólo pueden ser analizados hasta la profundidad que permite el conjunto de primitivos declarado ${ }^{6}$.

Dentro de una explicación es conveniente distinguir tres constituyentes:

(a) El primer constituyente es un conjunto que contiene los predicados clasificatorios de tipo más general (tales predicados son, por ejemplo, 'tipo natural', 'artefacto', 'vivo', 'cuerpo sólido', etc.). Como esta clasificación se efectúa en general con el empleo simultáneo de diferentes sistemas de clasificación, estos constituyentes constarán de varios elementos.

(b) El segundo constituyente es el conjunto de los predicados que guía la determinación de los correlatos (el uso del significante correspondiente). La consideración de un conjunto semejante no contradice la concepción de Putnam, es decir, la concepción de que el sentido no determina el correlato. Si bien el conocimiento de los no expertos sobre un correlato determinado puede ser falso, y tanto el saber de los no expertos como el de los expertos puede modificarse con el tiempo (y además también el saber de los expertos de un estadio de conocimiento anterior puede resultar falso), tanto las explicaciones especificas de no expertos como las específicas de expertos contienen siempre la descripción de propiedades cuya existencia constatable o no existencia en relación con un correlato dado para ella facilita la posibilidad de decisión sobre la pertenencia del correlato respectivo a una clase de correlato (con más o menos seguridad).

(c) El tercer constituyente representa, grosso modo, un conjunto de predicados complementarios ${ }^{7}$.

Puesto que cada explicación es un texto, estos tres constituyentes deben ser representados como constituyentes de este texto diferenciables entre sí.

1.4. Si favorecemos la validez/aplicabilidad del principio de Frege, debemos promover éstas a propósito de las representaciones de sentido relacionadas tanto con los explicandos como con los explicantes.

Esta promoción implica también que, si la buena formación semántico-intensional debe estar garantizada en el contexto normal, es conveniente asignar a las funciones de expresión las informaciones que

- En cuanto a la problemática de las explicaciones, cfr. por ejemplo Neubauer, 1980. Cfr. Kindermann, 1983 
aseguran las combinaciones semántico-intensionales admisibles. Esto puede suceder, por ejemplo, de modo que proporcionemos señales de selección a los puestos de constituyentes no ocupados en las estructuras fundamentales en el esquema 2, con las cuales el elemento que constituye la función de expresión figura en una relación de dependencia. (A (2) asignamos, por ejemplo, informaciones sobre a qué x puede ser aplicado el funtor 'corre' ('läuft'), y en relación con qué dimensión puede ser especificado.)

1.5. Si examinamos con profundidad consecuentemente los aspectos discutidos en los puntos 1.3 y 1.4, reconoceremos que la selección y estructuración de las funciones de expresión que han de ser almacenadas en el léxico representan una tarea sustancialmente compleja, como aparece de acuerdo con el análisis de la praxis lexicográfica.

La complejidad proviene, por una parte, de las relaciones que existen entre las explicaciones de las palabras de la lengua cotidiana y las de los mismos términos especializados en atención a sus formativos. Sin embargo, no trataré de estas cuestiones aquí ulteriormente.

La segunda fuente de la complejidad es la dificultad para definir el conjunto entero de los modismos reales y los cuasi-modismos. A este conjunto hay que asignarle no sólo las funciones de expresión cuya estructura de sentido no es isomorfa con la estructura sintáctica, sino también las funciones de expresión cuya significación puede, ciertamente, construirse a partir de la significación de sus constituyentes; no obstante, por la ocupación de puestos concretos de argumento de su parte de argumento a menudo puede entrar en consideración sólo un elemento o justamente pocos elementos. Tales funciones de expresión son (renunciando a la aplicación de representaciones formales), por ejemplo, las siguientes: ' $x$ vive (una) vida', ' $x$ sueña (un) sueño'; ' $x$ fuma algo'.

Atendiendo al hecho de que los constituyentes 'Leben' ('vida'), 'Traum' ('sueño'), 'Zigarette' ('cigarrillo'), 'Zigarre' ('cigarro'), etc. son combinables con especificadores de cualquier complejidad, no tendría sentido operar con funtores complejos tales como 'vive (una) vida', 'sueña (un) sueño', 'fuma (un) cigarrillo', 'fuma (un) cigarro', sino que los constituyentes 'vida', 'sueño', 'cigarrillo', 'cigarro' deben ser representados en los puestos de argumento correspondientes (de manera selectiva a través de la especificación de selección correspondiente).

A las funciones de expresión de mayor complejidad pertenecen además todas las funciones de expresión a partir de las cuales pueden ser realizadas las metáforas analizadas por Johnson y Lakoff".

\footnotetext{
* Cfr. Lakoff-Johnson, 1980.
} 


\section{Oraciones y sus significados}

2.0. Utilizo el término 'oración' exclusivamente como designación de un constructo teórico perteneciente al dominio de la gramática. Podemos decir de los dominios de la comunicación aquí considerados que la oración es el resultado de una abstracción por la que descuidamos los aspectos contextuales de un elemento de determinado orden de importancia de una comunicación real o potencial.

Tanto la buena formación sintáctica como la semántico-intensional del constructo llamado -en concordancia con la terminología tradicional- "oración simple», pueden ser definidas en la armazón de una gramática, mientras que, a propósito del constructo llamado "oración compuesta», en la armazón de una gramática sólo puede ser definida la buena formación sintáctica. A partir de las oraciones pueden construirse secuencias de oraciones; en relación con éstas podemos hablar de la conexidad (Konnexität) y de la cohesión (Kohäsion) de las secuencias de oraciones, así como de la buena formación sintáctica de cada uno de los constituyentes (en el caso de que un constituyente sea una oración simple, podemos hablar de la buena formación sintáctica y semántico-intensional). Doy por conocido el concepto de buena formación sintáctica y semántico-intensional de oraciones; de los conceptos de conexidad y cohesión me ocuparé en el punto 4.3.

2.1. Podemos hablar también de función de oración análogamente a la función de expresión. Una función de oración es en su forma mínima la configuración de dos funciones de expresión elementales, en la cual una representa una relación temporal y local no especificada y otra una especificación temporal y local. A partir de las funciones de oración mínimas construidas de este modo pueden producirse funciones de oración de cualquier complejidad, ya por incrustación vertical, ya por incrustación conectiva única o repetida. Con la utilización de esta función puede producirse la representación canónica de oraciones y secuencias de oraciones de cualquier complejidad.

2.2. De la anterior explicación del término 'oración' se sigue que el análisis semántico de oraciones simples está ante todo dirigido al análisis de su estructura de sentido. Este análisis significa distinguir si el sentido de la oración que ha de ser interpretada puede ser construido a partir del sentido de sus constituyentes de modo que nosotros (manteniéndonos en la armazón de un sistema de explicaciones) no infrinjamos en su caso las restricciones de selección dadas en el léxico, o determinemos cuántas y cuáles deben ser indispensablemente infringi- 
das en esta construcción. En este análisis ni debemos (a) operar incondicionalmente con los explicantes completos del sistema seleccionado de explicaciones de no expertos o de expertos, ni (b) debemos llegar hasta la representación de sentido compuesta exclusivamente de primitivos. (Aunque en el análisis también podemos operar con el indicador de correlato dado en el léxico, la configuración indicador-correlato es en el análisis de oración una estructura más o menos sin función.)

2.3. El análisis de la estructura de sentido de las oraciones compuestas y de las secuencias de oraciones sólo puede estar dirigido a la investigación de (a) si sus constituyentes-oraciones simples están sintáctica y semántico-intensionalmente bien formados, (b) si es posible desarrollar relaciones de sentido entre estos constituyentes con empleo del thesaurus general y especifico textual ( $y$ de las reglas de la gramática), y (c) si las oraciones compuestas y secuencias de oraciones son temáticamente bien formadas y dependientes del conjunto (cohesión). No podemos hablar de buena formación de la estructura de sentido de oraciones compuestas porque no es posible formular restricciones semántico-intensionales de selección en relación a los argumentos de los conectivos. Es válido de igual modo lo que concierne a la buena formación semántico-intensional de las secuencias de oraciones.

Si tenemos que analizar una oración o secuencia de oraciones en la que está contenida alguna palabra que no aparece como unidad en el thesaurus, no podremos ocuparnos del análisis de la estructura de sentido de esta oración/secuencia de oraciones en tanto que el thesaurus no esté completado con la unidad correspondiente a la palabra. Esta complementación no es, sin embargo, de tipo gramatical.

2.4. En el análisis semántico de oraciones y secuencias de oraciones no tiene sentido - -según la interpretación de estos términos anteriormente expuesta - hablar de valores de verdad. La definición de las llamadas relaciones de verdad tampoco puede ser considerada tarea de la gramática. Las oraciones no son ni verdaderas ni falsas. Análogamente, esto también es válido para las secuencias de oraciones.

\section{Actos comunicativos posibles y efectivos y sus significados}

3.0. 'Acto comunicativo' es la designación de una expresión perteneciente al dominio de la pragmática (y de la gramática). Desde el dominio de la gramática aquí considerado podemos decir que un acto comunicativo es la realización comunicativa potencial o efectiva de una oración. En analogía con las expresiones 'oración simple', 'oración 
compuesta' y 'secuencia de oraciones', podemos introducir las expresiones 'acto comunicativo simple', 'acto comunicativo compuesto' y 'secuencia de actos comunicativos' como designaciones de las realizaciones comunicativas (potenciales o efectivas) de los constructos gramaticales correspondientes. Si nos acercamos desde el dominio de la comunicación efectiva, podemos decir que un acto comunicativo posible es el resultado de una abstracción en la cual sustituimos los parámetros contextuales dados de un acto comunicativo efectivo por parámetros contextuales posibles.

3.1. La representación canónica de un acto comunicativo potencial o efectivo (o de una secuencia de actos comunicativos) es, en lo que concierne a su estructura, idéntica a la representación canónica de una oración (o de una secuencia de oraciones). En cuanto a su contenido de información, debe ser, sin embargo, de manera necesaria totalmente como aquélla, ya que su interpretabilidad también hace necesaria la consideración de la información que no se necesita en la interpretación de oraciones. Debemos investigar estas informaciones por via interpretativa, puesto que no están manifestadas en los actos comunicativos efectivos en la mayoría de los casos, por lo menos no lo están de modo claro y explicito. (Para estas informaciones cfr. los párrafos (2) y (3) del punto 3.4).

3.2. Puesto que todos los actos comunicativos potenciales y efectivos pueden ser analizados también como oraciones, su análisis sintáctico y semántico-intensional es realizado en su mayor parte en la armazón del análisis gramatical. El modificador "en su mayor parte" indica que dentro de cualquier teoría pragmática debe aclararse cómo pueden/deben ser definidos los aspectos semántico-intensionales y sintácticos específicos de acto comunicativo que pueden (o deben, oportunamente) ser analizados en la estructura de la gramática. Tales aspectos son, por ejemplo, los llamados aspectos específicos de registro, pues las informaciones especificas de registro, como es sabido, se manifiestan también morfológicamente en muchas lenguas.

En lo que sigue, en lugar de las expresiones 'análisis gramatical' y 'análisis pragmático' utilizaré 'análisis cotextual' y 'análisis contextual' (asi como las expresiones 'teoría cotextual' y 'teoria contextual', correspondientes a aquéllas), con lo que 'cotextual' y 'contextual' se refieren en su caso (potencial o efectivamente) al medio comunicativo de una expresión verbal. El empleo de esta terminología muestra ya de este modo que está motivado que un uso claro del término 'gramática' está relacionado problemáticamente con el nivel de oración; la estructura gramatical 
de una oración simple es propiamente 'gramatical' de manera diferente a la de una oración compuesta o de una secuencia de oraciones.

3.3. El análisis de los aspectos semióticos dominantes de los actos comunicativos debe tener lugar en el marco de una teoría contextual. Tal teoria debe proporcionar medios para el análisis de los aspectos siguientes: (a) relaciones de felicidad de los actos comunicativos concretos (qué acto es apropiado en qué contexto, en cumplimiento de qué relaciones, para qué funciones (para la realización de qué intenciones)); (b) relaciones de la buena formación, de la conexidad y de la cohesión de las diferentes secuencias de actos comunicativos (qué secuencias de qué actos comunicativos son apropiadas en qué contextos, en cumplimiento de qué relaciones, para qué funciones (para realización de qué intenciones)); (c) interpretación de los actos comunicativos/secuencias de actos comunicativos (en qué contexto, qué interpretaciones pueden ser aplicadas a actos y secuencias de actos (potenciales o efectivos) dados, en cumplimiento de qué relaciones, en uso de qué modelos de interpretación.

La distinción de actos comunicativos posibles y efectivos se basa en que en los actos posibles somos libres en lo que respecta a la construcción/postulación tanto de los contextos posibles como de las intenciones posibles, mientras que en los actos efectivos debemos atenernos a los contextos dados (o bien a las posibles interpretaciones de éstos).

3.4. A partir de los aspectos del análisis mencionados en el último punto querría hacer algunas consideraciones a propósito del aspecto de la interpretación.

(1) El concepto clave de la interpretación es el concepto de 'mundo real'. Éste no es un concepto constante que signifique lo mismo para todos los intérpretes. Por ello, la teoría de la interpretación debe permitir un manejo flexible de este concepto. El mundo real no significa para una persona o para una comuridad otra cosa que la totalidad de aquellos estados de cosas y de las relaciones entre ellos existentes que son/pueden ser considerados hechos por estas personas o por esta comunidad.

(2) La expresión 'acto comunicativo' también puede ser manejada flexiblemente. Todas las expresiones pueden ser reducidas a una de las siguientes tres modalidades performativas: (a) el hablante completa verbalmente una acción no exclusivamente verbal (cfr. por ejemplo un bautizo), (b) el hablante comunica algo (independientemente de que él tenga $o$ no un interlocutor real en el momento y en el lugar de la 
comunicación), (c) el hablante dice algo sin que se dé ninguno de los dos casos anteriores.

(3) En el caso de la comunicación es importante conocer la relación existente entre el estado de cosas comunicado, el hablante y el mundo considerado real por el hablante (por ejemplo, el hablante sabe, está convencido de, cree, etc. que el estado de cosas comunicado fue/es/será un estado de cosas en el mundo real).

(4) Los factores fundamentales arriba mencionados son aquellos con los que el intérprete debe operar en la construcción del modelo que sirve de base en la interpretación de un acto comunicativo y aun en la ejecución de la interpretación.

(5) La realización de la interpretación no sólo debe producir la clarificación del mundo en el que debe ser válido como estado de cosas lo comunicado y la clarificación de si es válido como tal en ese mundo (esto es, si esa comunicación es verdadera en relación con ese mundo); tal realización también significa la investigación sobre si el fragmento de mundo del hablante se aparta del fragmento de mundo del intérprete en opinión de éste, y si esta separación es motivada o no. (Si alguien comunica algo que no concierne a la 'realidad', esto todavía no significa que él quiera engañar a alguien con intención).

La meta de la interpretación de significado no es, en otras palabras, solamente averiguar interpretativamente el sentido de un objeto verbal producido en un contexto posible o efectivo y construir la representación de un correlato asignado interpretativamente a este sentido. Representa una tarea sustancialmente más compleja. Junto a la investigación interpretativa del sentido de un objeto verbal, el intérprete debe construir la representación de un correlato complejo, la cual contenga -en una unidad aceptable para el intérprete- los siguientes factores: (a) el mundo considerado real por el intérprete, (b) el mundo considerado real por el hablante según la opinión del intérprete, (c) el estado de cosas comunicado, como lo entiende el intérprete, (d) la relación entre el intérprete, el estado de cosas comunicado (como el intérprete lo entiende) y el mundo tenido por real por el intérprete, según el intérprete ve esta relación, (e) el estado de cosas comunicado, como lo comprende el hablante en opinión del intérprete, (f) la relación entre el hablante, el estado de cosas comuni cado (como lo entiende el hablante según opinión del intérprete) y el mundo considerado real por el hablante de acuerdo con la opinión del intérprete, como ve el intérprete esta relación, y finalmente (g) la relación entre las relaciones formuladas en (d) y (f), como las ve el intérprete ${ }^{9}$.

- Para estas cuestiones -ante todo en relación con textos_, cfr. Dorfmüller-Karpusa y Petöfi, 1981; Heydrich-Petöfi, 1981, y Petöfi, 1983a. La expresión 'correlato complejo' aquí 
(6) En el caso de la interpretación de secuencias de actos comunicativos el intérprete espera en general que la secuencia tenga cohesión y que los estados de cosas expresados en cada comunicación --análogamente a como el intérprete ve la relación entre estos estados de cosas y el mundo por él considerado real- constituyan una unidad coherente. Sin embargo, en este caso no se considera necesaria ninguna interpretabilidad (Interpretierbarkeit) que produzca un correlato acabado aceptable.

(7) En la interpretación de secuencias de actos comunicativos el thesaurus de la secuencia que ha de interpretarse debe ser construido como constituyente de la interpretación que se produzca. Este thesaurus debe adjudicar a cada presencia de cada uno de los formativos la explicación del sentido que ha de manifestarse (mejor, motivarse) con la mayor probabilidad en el cotexto (y contexto) dado. Estas explicaciones de sentido son configuraciones parciales de las explicaciones del thesaurus general primitivo y/o ampliado o son parcial o totalmente idiosincráticas.

\section{Textos y sus significados}

4.0. En cuanto al empleo del término 'texto', no considero la 'textualidad' una propiedad inherente de los objetos verbales. Para un intérprete, una secuencia de actos comunicativos posibles o efectivos es un texto si cumple las condiciones funcionales que él considera relevantes. En relación con las secuencias de oraciones no considero significativo el empleo de la designación 'texto'. Dependiendo de que el intérprete considere texto una secuencia de actos comunicativos posibles o efectivos, podemos hablar de textos posibles y de textos efectivos.

Las condiciones funcionales relevantes pueden ser totalmente formales; por ejemplo, la condición de que un objeto verbal incluido en un volumen con el título Narraciones sea un objeto verbal independiente claramente delimitable de todos los demás objetos verbales independientes publicados en este volumen. (Puede aceptarse que los intérpretes puedan constatar, en conformidad, en los contextos respectivos lo que se considera objeto verbal independiente). Esta delimitabilidad

utilizada no es idéntica al término 'correlato' usado para indicar un componente del triángulo semiótico. Para evitar equívocos - relativos también a las expresiones 'mundo' y 'mundo textual' - a partir de ahora se utilizará 'relato' (relatum) y 'universo de relato' (relatumuniverse) en lugar de 'mundo textual', e 'interpretamento' (interpretamentum) en lugar de 'correlato complejo'. Cfr., por ejemplo, Petöfi-Sözer, 1984. 
puede en adelante ser tan 'evidente' que la calificación como texto del objeto en cuestión no haga preciso que se deba tratar primero a fondo dicho objeto.

La consideración de un objeto como texto implica determinadas expectativas convencionalizadas. La más decisiva de éstas es la de que este objeto representa, en un determinado sentido, un 'conjunto íntegro', es decir, que la configuración de estado de cosas que se le puede asignar como correlato en sentido estricto es un fragmento continuo de la realidad considerable como íntegro en sí.

4.1. Todos los objetos considerados textos pueden, no obstante, ser analizados como secuencias de actos comunicativos y como secuencias de oraciones. Todo lo que puede decirse en relación con el análisis de secuencias de oraciones y actos comunicativos puede también ser dicho con respecto al análisis de un objeto verbal considerado como texto. La representación canónica de un objeto verbal considerado como texto tampoco se distingue de la representación canónica de una secuencia de actos comunicativos.

4.2. En atención a la interpretación de significado de textos y de secuencias de actos comunicativos, junto a las semejanzas hay también diferencias sustanciales. $Y$ más importante, aparece la distinción que resulta de la expectativa de 'integridad' en relación con los textos. En tanto que consideramos un objeto verbal como una secuencia de actos comunicativos, esta secuencia no es propiamente otra cosa que el conjunto ordenado de sus constituyentes, y su interpretación se realiza mediante la reunión de las interpretaciones de sus constituyentes. En tanto que consideramos un objeto verbal como texto, sus constituyentes se hacen constituyentes de una 'totalidad' (tomada en un sentido determinado); la meta de su interpretabilidad llega a ser la construcción/reconstrucción de una 'configuración de estado de cosas considerable en si como totalidad', y la interpretación de los elementos concretos de la secuencia es motivada y/o determinada implícita o explícitamente por esta configuración tenida en cuenta aquí.

4.3. Se hace evidente, a partir de la discusión hasta ahora desarrollada de la interpretación de significado de objetos verbales de diferentes tipos y de complejidad varia, que las cuatro propiedades siguientes de los objetos verbales son para mí las más importantes: conexidad, cohesión, coherencia (Kohärenz) e interpretabilidad. En lo que sigue me gustaria ocuparme de estas propiedades -utilizando un sencillo esquema (cfr. esquema 4). 
4.3.1. Llamo 'interpretable' a un objeto considerado como texto si se logra asignar a este objeto un correlato tomado en sentido estricto que represente para el intérprete una configuración continua del estado de cosas observable como totalidad. (Esta configuración de estado de cosas es la parte (a) en el esquema 4 ; los símbolos «K $\mathrm{K}_{\mathrm{a}}$ ",.. « « $\mathrm{K}_{\mathrm{i}}$ " remiten a los nueve correlatos (estados de cosas) que son los constituyentes de esta configuración; las líneas dobles enlazan entre sí, de dos en dos, correlatos continuos.

4.3.2. Supongamos que falta la manifestación del correlato $K_{f}$ en el texto que va a ser interpretado. Si el texto contuviera la manifestación de los nueve correlatos (y sólo de éstos), el texto podría ser considerado "coherente» e «interpretable», suponiendo que esté bien formado cotextualmente. Cuando no es éste el caso, el texto no es coherente. No obstante, por falta de coherencia no se sigue todavia que el texto no sea interpretable. Si el intérprete consigue derivar motivadamente la representación de $\mathrm{K}_{\mathrm{f}}$ de la representación de los otros ocho correlatos representados en el texto (en otras palabras, si consigue completar el texto con la representación de $\mathrm{K}_{\mathrm{f}}$ de una manera motivada), podría aquél llamar verdaderamente al texto no "coherente», sino «interpretable». De aquí se sigue que la coherencia no es una condición necesaria de la interpretabilidad. Si en el texto faltara la manifestación de $K_{i}$, y sin embargo los otros correlatos (y sólo éstos) estuvieran contenidos, entonces el texto podría ser llamado en verdad "coherente», pero no «interpretable». El texto llegaría a ser interpretable solamente si pudiera ser completado por medio de la manifestación de $\mathrm{K}_{i}$ de manera motivada. Se sigue de esto que la coherencia tampoco es una condición suficiente de la interpretabilidad. (Estos dos casos están representados en el esquema 4 como (b1) y (b2)).

4.3.3. Mientras la coherencia y la interpretabilidad no son propiedades de un objeto verbal descubribles de modo intersubjetivo, sino propiedades que le son asignadas (o no) por medio de un intérprete dado con utilización de una teoría de interpretación dada y de un modelo de interpretación dado, la conexidad puede ser considerada como una propiedad de un objeto verbal descubrible intersubjetivamente, incluso si el descubrimiento de esta propiedad depende de una teoria (pero no de un intérprete y de un modelo de interpretación).

La conexidad es - localmente tenida en cuenta - una relación de enlace, respectivamente, entre dos constituyentes independientes de una secuencia de oraciones, de una secuencia de actos comunicativos o de un texto, sostenida de manera explicita por algunos elementos de 


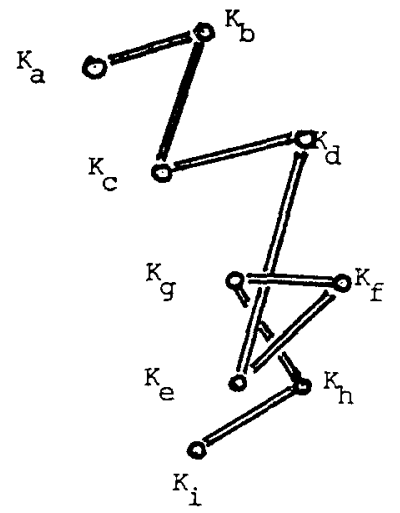

(4) (a)

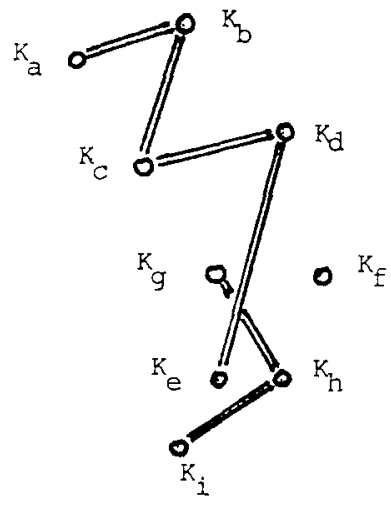

(4) (b1)

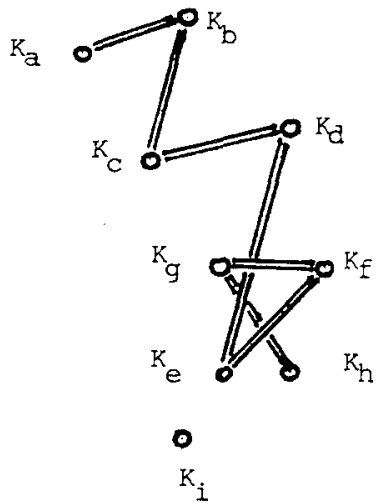

(4) (b2)
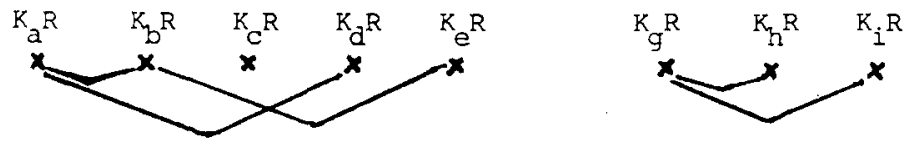

(4) (c)

Esquema 4.-Configuraciones de correlatos y representaciones de correlato. 
estos objetos verbales. Secuencias de oraciones, secuencias de actos comunicativos o textos -tomados en consideración globalmenteson conexos si no contienen constituyentes que no estén enlazados con otros constituyentes. Con esto no se exige que los constituyentes contiguos estén enlazados unos con otros, antes bien, es importante que no contengan constituyentes solos aislados de los demás. (Una operación típica creadora de conexidad es la sustitución pronominal y/o léxica). Hay que comprender fácilmente que la conexidad no es una condición necesaria ni suficiente de la coherencia y de la interpretabilidad. Tampoco es una condición suficiente de la buena formación cotextual (cfr. 4.3.4.).

El descubrimiento de la coherencia y su descripción, así como la ejecución de la interpretación son tareas contextuales. El descubrimiento de la conexidad es tarea exclusivamente cotextual, o cotextual $y$ contextual, dependiendo de que, por ejemplo, los registros sean vistos como pertenecientes al dominio cotextual o al contextual. Si los consideramos fenómenos contextuales, pueden darse entonces los casos siguientes: una secuencia de actos comunicativos está conectada cotextualmente, pero no contextualmente, o viceversa, con lo que descuidamos los otros dos casos igualmente posibles. En otras palabras, esto significa que debemos distinguir aquí entre conexidad cotextual y contextual.

4.3.4. La conexidad todavia no garantiza en sí la buena formación cotextual de un objeto verbal (de una secuencia de oraciones o actos comunicativos, o de un texto). Incluso en el caso de que los constituyentes de un objeto verbal estén bien formados y el objeto verbal sea conexo, puede éste ser contextualmente no bien formado. Es éste el caso, por ejemplo, si (a) la estructura lineal tema-rema de los constituyentes particulares es tal que el objeto verbal no puede ser considerado continuo en vista de la progresión temática y/o remática, o (b) si el objeto verbal no puede ser visto en el nivel macrotemático como un objeto que constituye una totalidad. Si un objeto verbal cumple todas las condiciones de la buena formación cotextual, es llamado cohesivo, mientras que la propiedad de la buena formación cotextual es denominada cohesión.

El descubrimiento y descripción de la cohesión son - de manera semejante al descubrimiento y descripción de la conexidad, y por el mismo fundamento - una tarea exclusivamente cotextual o una tarea cotextual $y$ contextual.

La conexidad es una condición necesaria pero no suficiente de la cohesión; la cohesión no es — de modo semejante a la conexidad- 
una condición necesaria ni suficiente de la coherencia. Ciertamente, un texto puede, por ejemplo, ser cohesivo (expresado de otro modo, puede constituir una unidad continua semántico-intensionalmente y también bien formada en el aspecto macrotemático), pero puede ser discrepante semántico-intensionalmente de tal modo que un intérprete dado puede ser incapaz de asignar al texto una interpretación figurativa que le permita la construcción de un correlato aceptable y puede por lo tanto considerar este texto no coherente. Más exactamente, debería decirse que no tiene sentido alguno para él la cuestión de si este texto es o no coherente ${ }^{10}$.

\section{Consideraciones finales}

Me he ocupado en el presente artículo de algunos aspectos generales de la interpretación de significado. No obstante, no he examinado todas las cuestiones importantes, así, por ejemplo, no he tratado la relación entre la interpretación literal, la figurativa y la simbólica, sin la cual es inimaginable que se haya clarificado una interpretación de significado adecuada ". Como conclusión quiero referirme a los puntos siguientes:

(1) La interpretación del significado de la palabra, de oraciones, de actos comunicativos y secuencias de actos comunicativos y de textos sería impensable sin la consideración de factores psicológicos y sociológicos (etnometodológicos) fundamentales. En la construcción de una teoría del significado (una teoría de la interpretación del significado) adecuada deben ser tomados en consideración, por una parte, los resultados de las disciplinas particulares de este ámbito multidisciplinar de investigación $y$, por otra, los problemas metodológicos resultantes dentro de cada una de las disciplinas y a partir de la cooperación de estas disciplinas.

(2) Ya que la coherencia (en la concepción discutida en este artículo) está estrechamente ligada con la interpretabilidad, no puede tener lugar una adecuada discusión de la coherencia sin haber analizado antes apropiadamente los aspectos de la interpretación. De lo contrario, puede aplicarse a lo sumo a cuestiones de la conexidad y de la cohesión (según han sido entendidas en este artículo), que ciertamente

10 Vid. Hatakeyama-Petöfi-Sözer, 1984 a propósito del tema de la conexidad, cohesión, coherencia e interpretabilidad.

"En cuanto a la interpretación figurativa, vid. Johnson, 1981; Ortony, 1979, y Petöfi, $1983 b$. 
no son cuestiones irrelevantes, pero a propósito de la comunicación sólo poseen relevancia en relación con la coherencia.

(3) En consideración a la compleja naturaleza del análisis de objetos verbales - sobre qué plano debe éste también ser conducido-, considero indispensable emplear una lengua canónica. La estructura de esta lengua canónica debe ser independiente de las particularidades individuales de las lenguas naturales y debe garantizar la máxima flexibilidad. Tanto la ejecución de la interpretación y el análisis de la coherencia como el análisis y descripción de aquellas propiedades de los objetos verbales relacionadas con la llamada estructura de superficie son claramente posibles sólo con ayuda de una lengua canónica.

(Traducido del alemán por Tomás Albaladejo)

\section{Referencias bibliográficas}

Biasci, C.-J. Fritsche (eds.) (1978), Texttheorie, Textrepräsentation. Theoretischen Grundlagen der kanonischen sinnsemantischen Repräsentation von Texten, Hamburgo, Buske.

Dorfmüller-Karpusa, K., Petöfi, J. S. (1981), Text, Kontext, Interpretation. Einige Aspekte der textheoretischen Forschung, Hamburgo, Buske.

Eikmeyer, H.-J., Rieser, H. (eds) (1981), Words, Worlds, and Context. New Approaches in Word Semantics, Berlín-Nueva York, De Gruyter.

Hatakeyama, K., Petöfi, J. S., Sözer, E. (1984), "Text, Konnexität, Kohäsion, Kohärenz", en: M.-E. Conte (ed.), Kontinuität und Diskontinuität in Texten und Sachverhaltskonfigurationen. Diskussion über Konnexität, Kohäsion und Kohärenz, Hamburgo, Buske, 1984. (Aparece en inglés en: E. Sözer (ed.), Text Connexity, Text Coherence. Aspects, Methods, Results, Hamburgo, Buske, 1984.)

Heydrich, W., Petöfi, J. S. (1981), «Pragmatic Considerations Within a Text-theoreticall Framework", en: H. Parret, M. Sbisa y J. Verschueren (eds.), Possibilities and Limitations of Pragmatics, Amsterdam, John Benjamins, 1981, pp. 313-330.

- (1983), «A Text-theoretical Account of Questions of Lexical Structure», en: Quaderni di Semantica, 4, 1: pp. 120-127, 2: pp. 66-76. 
Johnson, M. (ed.) (1981), Philosophical Perspectives on Metaphor, Minneapolis, University of Minnesota Press.

Kindermann, J. (1984), "Wortgesteuertes Parsing. Überlegungen zu Struktur und Prozedur», en: A. Rothkegel, B. Sandig, B. Weissgerber (eds.), Text - Textsorten - Semantik, Hamburgo, Buske.

Lakoff, G., Johnson, M. (1980), Metaphors We Live By, ChicagoLondres, The University of Chicago Press.

Neubauer, F. (1980), Die Struktur der Explikationen in deutschen einsprachigen Wörterbüchern. Eine vergleichende lexikosemantische Analyse, Hamburgo, Buske.

- Petöfi, J. S., «Word Semantics, Lexicon Systems, and Text Interpretation», en: Eikmeyer, Rieser (eds.) (1981), pp. 343-377.

Ortony, A. (ed.) (1979), Metaphor and Thought, Cambridge, Cambridge University Press.

Petöfi, J. S. (1979a), «La teoría lógico-semántica de las lenguas naturales como teoria textual», en: Petöfi, García Berrio (1979), pp. 99- 125.

- (1979b), «Una teoría textual formal y semiótica como teoría integrada del lenguaje natural», en Petöfi, García Berrio (1979), pp. 127-145.

- (1979c), «Estructura y función del componente gramatical de la teoria de la estructura del texto y de la estructura del mundo», en Petöfi, García Berrio (1979), pp. 147-189.

- (1979d), "Léxico, conocimiento enciclopédico, teoria del texto", en: Petöfi, Garcia Berrio (1979), pp. 191-213.

- (1979e), «La representación del texto y el léxico como red semántica», en: Petöfi, García Berrio (1979), pp. 215-242.

- (1980), «Representation Languages and their Function in Text Interpretation", en: S. Allén (ed.), Text Processing, Aspects of Text Analysis and Generation, Text Typology and Attribution, Proceedings of Nobel Symposium 51, 1980, Stockholm, Estocolmo, Almquist and Wiksell International, 1983, pp. 85-122.

- (1983), "Text, Signification, Models and Correlates. Some Aspects of Text Comprehension and Text Interpretation», en: G. Rickheit, M. Bock (eds.), Psycholinguistic Studies in Language Processing, Berlín-Nueva York, De Gruyter, 1983, pp. 266-298.

- (1984), "Le metafore nella comunicazione quotidiana e nei testi scientifici, biblici e letterari", en: Interpretazione e simbolo. Atti del $V$ Colloquio sulla Interpretazione, Macerata, 21-22 marzo 1983, Turín, Marietti, 1984, pp. 97-132.

- García Berrio, A. (1979), Lingüistica del texto y critica literaria, Madrid, Comunicación. 
- Sözer, E. (1984), «Static and Dynamic Aspects of Text Constitution», en: J. S. Petöfi (ed.), Text and Dircourse Composition. Empirical Aspects, Theoretical Approaches, Berlín - Nueva York.

Putnam, H. (1978), «Meaning, Reference and Stereotypes», en: F. Guenthner, M. Guenthner-Reutter (eds.), Meaning and Translation. Philosophical and Linguistic Approaches, Londres, Duckworth, 1978, pp. 61-81.

Stegmüller, W. (1979), Hauptsrömungen der Gegenwartsphilosophie, vol. II, 6. ${ }^{a}$ ed. ampliada, Stuttgart, Kröner. 